CASTROVIEJO, S. et al. Flora Iberica, Vol. $\mathrm{X}: 331-335$.

TALAVERA, S. y B. VALDÉS -1976- Revisión del género Cirsium (Compositae) en la Península Ibérica. Lagascalia 5 (2): 127-223.

VALDÉS, B., M. REJDALI, A. ACHHAL EL KADMIRI, J. L. JURY \& J. M. MONSERRAT (eds.) -2002-Catalogue des Plantes Vasculaires du nord Maroc, incluant des clés d'identification. Checklist of Vascular Plants of $N$ Morocco with identification keys. Madrid: Consejo Superior de Investigaciones Científicas.
VALDÉS, B., S. TALAVERA y E. FERNÁNDEZ-GALIANO (eds.) -1987Flora Vascular de Andalucía Occidental. Vols. I-III. Barcelona: Ketres Editora, s. a.

Aceptado para su publicación en octubre de 2005

Dirección de los autores. Depto. de Biología Vegetal, Facultad de Ciencias, Universidad de Málaga, apdo. 59, E-29080 Málaga, España. Email:ogavira@uma.es

\title{
103. DATOS SOBRE LA FLORA Y VEGETACIÓN DE SIERRA PRIETA (SERRANÍA DE RONDA, MÁLAGA, ESPAÑA)
}

Baltasar CABEZUDO, Oscar GAVIRA y Andrés V. PÉREZ LATORRE

New data on the flora and vegetation of Sierra Prieta mountain range (Serrania de Ronda, Malaga, Spain)

Palabras clave. Flora, asociaciones vegetales, Ronda, Málaga, España.

Key words. Flora, plant communities, Ronda, Malaga, Spain.

La tradicional atracción por la Sierra de las Nieves y sus pinsapares ha hecho que pocos botánicos hayan recolectado en zonas próximas al núcleo central de dicha sierra (Ceballos y Vicioso, 1933; Pérez Latorre et al., 1998). Con el fin de colaborar con varios proyectos actuales sobre flora española y andaluza hemos iniciado las recolecciones en alguna de estas zonas poco visitadas y queremos dar a conocer su vegetación y sus elementos florísticos más característicos. En esta nota, se presentan los resultados de las herborizaciones e inventarios en Sierra Prieta (Casarabonela, El Burgo, Alozaina y Ardales, provincia de Málaga), todos ellos en la cuadrícula 30 SUF37.

El complejo montañoso donde se incluye Sierra Prieta está situado en la provincia de Málaga, en la comarca de la Serranía de Ronda ocupando términos municipales de Alozaina, Casarabonela, El Burgo y Yunquera. Topográficamente se trata 
de una montaña aislada, la segunda más alta de la Serranía de Ronda malagueña con 1520 m. Está dividida en dos partes: Sierra Prieta, objeto de este estudio, y Sierra Cabrilla al NW, separadas por un claro puerto entre ambas.

Litológicamente está constituida básicamente por calizas y margas del Jurásico y dolomías (a veces con grado de kakiritización) del Triásico de la Unidad de las Nieves, con dominio de litosoles en las zonas rocosas y canchales y por entisoles en el resto, debido a la dureza de la roca, a las fuertes pendientes y a la profunda erosión por la deforestación sufrida. En algunas zonas más llanas o con vegetación conservada se pueden encontrar inceptisoles, capaces de soportar bosques climácicos. No existen cursos permanentes de agua, tan solo nacimientos tobáceos, a veces muy importantes, en la base de la sierra.

La bioclimatología de la Sierra se ha estudiado en base a fitoindicadores, ya que sólo existe en la zona la estación de Casarabonela ( $\mathrm{P}$ media anual $823 \mathrm{~mm}, \mathrm{~T}$ media anual $16,4^{\circ} \mathrm{C}$, altitud $494 \mathrm{~m}$., $\mathrm{It}=425$.) que se corresponde con un piso bioclimático termomediterráneo y ombrotipo subhúmedo superior. Situamos los límites de este piso entre los $400 \mathrm{~m}$. (base de la sierra) y los 800 m. Hemos reconocido el piso mesomediterráneo, aproximadamente y variando entre solana y umbría, entre los $600 / 900$ a 1300/1400 m. y el ombrotipo, según el aumento de precipitación debido a la altitud observado en la cercana Sierra de las Nieves, debe de estar en el límite entre el subhúmedo superior y el húmedo inferior. También se reconoce el piso supramediterráneo inferior, con variación altitudinal solana-umbría, entre 1200/1400 a $1520 \mathrm{~m}$. y un ombrotipo húmedo inferior (1000-1300 mm. estimado).

Biogeográficamente la Sierra Prieta se incluye en la parte más oriental del subsector
Rondense (sector Rondeño, provincia Bética, superprovincia Iberomarroquí-Atlántica, subregión Mediterránea Occidental).

El estudio de la vegetación se ha realizado utilizando el método fitosociológico, las series de vegetación y el concepto de zonopotencialidad (Pérez Latorre et al., 2004). Los resultados obtenidos se agrupan por pisos bioclimáticos.

\section{Piso termomediterráneo (300-800 m)}

La zonopotencialidad corresponde a encinares termófilos de Smilaci-Querceto rotundifoliae $S$ en su faciación basófila. Dichos encinares han desaparecido en su totalidad y han sido sustituidos por matorrales seriales de Cisto clusii-Ulicetum baetici thymetosum capitati o, en lugares repetidamente incendiados y pastoreados, por una comunidad basal de Cistus albidus. En la base de algunos barrancos es posible observar el coscojar de Rhamno-Quercetum cocciferae con abundante Pistacia terebinthus, que constituye la vegetación climácica sobre dolomías en zonas abruptas, o con Ononis speciosa y Anthyllis cytisoides en taludes arcillosos. También son frecuentes enebrales de Juniperus oxycedrus. El paisaje vegetal se completa con repoblaciones de Pinus halepensis.

La vegetación exoserial está representada por una comunidad basal de Putoria calabrica en los roquedos soleados. Suelos alterados y en cierto modo nitrificados y profundos presentan puntualmente una singular comunidad de Salvia candelabrum.

Los escasos barrancos que reciben algún aporte hídrico presentan adelfares de RuboNerietum oleandri.

\section{Piso mesomediterráneo (800-1300 m)}

La zonopotencialidad depende en gran medida de la topografía y la orientación. 
a/ en la vertiente sur y oeste, corresponde a encinares basófilos de Paeonio-Querceto rotundifoliae $S$ (faciación pistacietoso $\boldsymbol{s}$ hasta los $1000 \mathrm{~m}$.), sobre suelos zonales, mientras que sobre kakiritas, resaltes rocosos y laderas de muy fuerte pendiente corresponde a sabinares con pinos carrascos de Abieto-Junipereto phoeniceae pinetoso halepensis $\boldsymbol{S}$. Los encinares han desaparecido prácticamente debido a los incendios recurrentes aunque es posible reconocer puntualmente y en regeneración el coscojar (RhamnoQuercetum cocciferae) de orla del encinar. Los sabinares se han refugiado en zonas protegidas de los incendios, como crestas y espolones rocosos, quedando en algunos lugares solo Pinus halepensis procedente de regeneración. El matorral es común a ambos y corresponde a Ulici-Lavanduletum lanatae, que, sobre dolomías y kakiritas, lleva en los claros el tomillar hiperxerófilo de GalioThymetum granatensis y en zonas calizas de suelo medianamente erosionado a Stipa gigantea; en el tránsito al piso supramediterráneo es abundante Festuca scariosa, mientras que hacia zonas inferiores lo es Stipa tenacissima. Hay zonas del matorral donde el incendio ocurrió menos recientemente y se halla en mosaico con fenalares de Phlomido-Brachypodietum retusi con abundante Ptilostemon hispanicus.

La vegetación exoserial esta ligada a los roquedos aislados por el abundante matorral. Se presenta una comunidad basal de Putoria calabrica en rocas soleadas, mientras que, en grietas más umbrías, aparece una comunidad basal de Hieracium baeticum. En las gleras se desarrollan comunidades de Crambe filiformis en el horizonte inferior y de Sedum sediforme en los horizontes medio y superior.

b/ en la vertiente norte y este la zonopotencialidad corresponde a encinares con quejigos de Paeonio-Querceto rotundifoliae quercetoso fagineae s, aunque en los espolones, roquedos, canchales y farallones, con litosuelos, se encuentran sabinares de Abieto-Junipereto phoeniceae $\boldsymbol{S}$. En las vaguadas y umbrías con suelos más profundos, por encima de $1200 \mathrm{~m}$. (mesomediterráneo superior) la zonopotencialidad corresponde a acerales de Daphno-Acereto granatensis $\boldsymbol{S}$; sus escasos bosquetes relictos están orlados por incipientes espinares caducifolios de PrunoBerberidetum hispanicae. Gran parte de la zona está repoblada con Pinus halepensis y Pinus nigra. El matorral también pertenece a Ulici-Lavanduletum lanatae (subasociación erinaceetosum a partir de 1200 m.), con Galio-Thymetum granatensis solo en zonopotencialidad de los sabinares (Abieto-Juniperetum phoeniceae). Se detecta una incipiente comunidad basal de Erinacea anthyllis (piornales xeroacánticos) en la serie de los acerales a unos 1300 m., ya en el límite inferior del piso supramediterráneo.

La vegetación exoserial corresponde a la asociación rupícola esciófila de RhamnoSaxifragetum granatensis. En las oquedades sombreadas, se ha detectado el Stachydetum circinatae. En los canchales fijos se desarrolla una comunidad basal de Rumex induratus.

\section{Piso supramediterráneo (1300/1400-1500 m)}

a/ las vertientes sur y oeste están dominadas por matorrales cuya composición y estructura depende de los incendios recurrentes. El matorral es de UliciLavanduletum lanatae erinaceetosum, sobre todo en dolomías, ya que sobre calizas el matorral es poco diverso, dominando Ulex baeticus y Cistus albidus. En litosoles dolomíticos, en la zonopotencialidad de Abieto-Juniperetum phoeniceae, aparecen buenas representaciones del GalioThymetum granatensis. 
b/ las vertientes norte y este están ocupadas casi totalmente por extensos farallones rocosos y canchales de unos 150$200 \mathrm{~m}$ de desnivel, donde la zonopotencialidad corresponde a complejos edafogénicos rupícolas, con un mosaico de especies climácicas de los encinares y de los acerales en mezcla con sabinares, dominando las comunidades rupícolas (RhamnoSaxifragetum granatensis) y subrupícolas (comunidad basal de Rumex induratus). Los mejores sabinares de Abieto-Juniperetum phoeniceae se encuentran a partir de 1300 $\mathrm{m}$. en orientación este sobre un gran espolón rocoso que parte de la cumbre de Sierra Prieta y llega prácticamente a la base de la sierra. En las umbrías, sobre entisoles fuera de zonas rocosas, en la zonopotencialidad del Daphno-Acereto granatensis $\boldsymbol{S}$, aparece un piornal xeroacántico de la comunidad basal de Erinacea anthyllis, en mosaico con tomillares de Galio-Thymetum granatensis indicando dolomías, o con un lastonar de Festucion scariosae indicando suelos conservados. En esa zona además podemos encontrar una comunidad basal de Crataegus monogyna y Prunus mahaleb (existen repoblaciones de Abies pinsapo y Pinus nigra).

Como vegetación exoserial es destacable la presencia, en pavimentos rocosos umbríos por encima de $1450 \mathrm{~m}$., de pastizales psicroxerófilos de la comunidad basal de Erodium cheilanthifolium y Poa ligulata.

Respecto al listado de flora recolectada, centrada en los pisos meso y supramediterráneo, para cada taxon se señala su nombre y autor(es), municipio, altitud, pliego MGC, adscripción fitosociológica y categoría en la Lista Roja Andaluza (en su caso). El material herborizado se encuentra depositado el Herbario de la Universidad de Málaga (MGC).
Acer opalus subsp. granatense (Boiss.) Font Quer \& Rothm.

El Burgo. 1160 m. MGC 61952. AceriQuercion fagineae. NT

Acinos alpinus (L.) Moench

El Burgo. 1160 m. MGC 61954

Anthemis tuberculata Boiss.

Casarabonela. 1100 m. MGC 61982

Anthyllis podocephala Boiss.

Casarabonela. 900 m. MGC 61907.

Campanulion velutinae.

Arenaria erinacea Boiss.

Casarabonela. 1050-1500 m. MGC 63028, 61559, 61968, 61977. El Burgo. 1260 m. MGC 61933. Andryalion agardhii.

Ballota hirsuta Benth. subsp. hirsuta

Casarabonela. 960 m. MGC 61981.

Brassica repanda subsp. latisiliqua (Boiss. \& Reut.) Heywood

El Burgo. 1260 m. MGC 61935.

Calamintha nepeta (L.) Savi

Casarabonela. 800 m. MGC 63027. Rubo-

Nerion oleandri.

Celtis australis L.

Alozaina. 860 m. MGC 63032. NT

Centaurea prolongoi Boiss. ex DC.

Alozaina. $1350 \mathrm{~m}$. MGC 61927.

Casarabonela. 960-1070 m. MGC 61958, 61978.

Campanulion velutinae. VU

Centaurium erythraea Rafn.

Casarabonela. 900 m. MGC 61967.

Clematis vitalba $\mathrm{L}$.

El Burgo. 1170 m. MGC 61922. LoniceroBerberidion hispanicae.

Crambe filiformis Jacq.

Alozaina. 1350 m. MGC 61926. AndryaloCrambion filiformis. 
Crepis albida Vill. subsp. albida

Casarabonela. 1100 m. MGC 61983. Campanulion velutinae.

\section{Daphne laureola L.}

El Burgo. 1160 m. MGC 61956. AceriQuercion fagineae.

Dianthus boissieri Willk.

Casarabonela. 900 m. MGC 61986. Campanulion velutinae.

Digitalis laciniata Lindl. subsp. laciniata

Casarabonela. 900 m. MGC 61931. Lavandulo-Echinospartion boissieri. DD

Ephedra fragilis Desf.

Alozaina. 850 m. MGC 63031

Erodium cheilanthifolium Boiss.

Casarabonela. 1300-1450 m. MGC 63033. Koelerio-Sedetea

Festuca capillifolia Dufour

El Burgo. 1250 m. MGC 61944.

Ficus carica L.

El Burgo. 1170 m. MGC61921.

Fumana ericifolia Wallr.

Casarabonela. 1050 m. MGC 62079. Lavandulo-Echinospartion boissieri.

Galium baeticum (Rouy) Ehrend. \& Krendl

Alozaina. $1350 \mathrm{~m}$. MGC 61928. Casarabonela. 1100 m. MGC 61965. Andryalion agardhii. VU

\section{Helleborus foetidus L.}

E1 Burgo. 1160 m. MGC 61951. AceriQuercion fagineae.

\section{Helichrysum stoechas (L.) Moench}

Casarabonela. 1070-1400 m. MGC 61957, 63026.

Iberis pectinata Boiss. \& Reuter Casarabonela. 1320 m. MGC 61969.

Inula montana $\mathrm{L}$.
Casarabonela. 1100 m. MGC 61963.

Jasione foliosa Cav.

Casarabonela. 1350 m. MGC 61975.

Saxifragion camposii.

Juniperus phoenicea L.

Casarabonela. 1070-1300 m. MGC 61964, 63030. Pino-Juniperion phoeniceae. NT

Jurinea pinnata (Lag.) DC.

Casarabonela. 990 m. MGC 61960, 61973. Andryalion agardhii.

Klasea pinnatifida (Cav.) Cass.

Alozaina. 1350 m. MGC 61929-61980.

Koeleria vallesiana (Honckeny) Gaudin

Casarabonela. 990 m. MGC 61971.

Festucion scariosae.

Leuzea conifera (L.) DC.

Casarabonela. 1100 m. MGC 61984.

Lonicera etrusca G. Santi

El Burgo. 1180 m. MGC 61946. Lonicero-

Berberidion hispanicae.

Odontites longifolia (Vahl) Webb

El Burgo. 1250 m. MGC 61942.

Ononis speciosa Lag.

Casarabonela. 990 m. MGC 61910. Rhamno-Quercion cocciferae.

Orobanche haenseleri Reut.

El Burgo. 1180 m. MGC 61949. AceriQuercion fagineae. DD

Petrorhagia saxifraga (L.) Link

Alozaina. 1100, 1350 m. MGC 61954, 61925. El Burgo. 1180 m. MGC 61936.

Saxifragion camposii. DD

Phlomis x composita Pau

El Burgo. 1250 m. MGC 61917. LavanduloEchinospartion boissieri.

Phlomis crinita subsp. malacitana (Pau) Cabezudo, Nieto \& T. Navarro 
Casarabonela. 1100 m. MGC 61918. Lavandulo-Echinospartion boissieri.

Pimpinella tragium subsp. lithophila (Schischk.)

Tutin

Casarabonela. 1320 m. MGC 61970. El Burgo. 1260 m. MGC 61934. Andryalion agardhii.

Pinus nigra Arnold 63029 .

El Burgo. 1250-1450 m. MGC 61939,

Pleurosorus hispanicus (Cosson) Mett.

E1 Burgo. 1100 m. MGC 61938. Saxifragion camposii.

Potentilla caulescens L.

Casarabonela. 1100 m. MGC 61962.

Saxifragion camposii.

Prunus mahaleb L.

El Burgo. 1170 m. MGC 61920. LoniceroBerberidion hispanicae.

Prunus ramburii Boiss.

El Burgo. 1250 m. MGC 61940. LoniceroBerberidion hispanicae. VU

Reseda undata subsp. gayana (Boiss.) Valdés Berm.

Casarabonela. 960 m. MGC 61979. DD

Rosa micrantha Borrer ex Sm.

E1 Burgo. 1160-1170 m. MGC 61919. Lonicero-Berberidion hispanicae.

Rumex induratus Boiss. \& Reuter

E1 Burgo. 1180 m. MGC 61948. Rumicetalia indurati.

Santolina rosmarinifolia $\mathrm{L}$.

El Burgo. 1250 m. MGC 61941.

Saxifraga globulifera Desf.

El Burgo. 1100 m. MGC 61937. Saxifragion camposii.

Scabiosa turolensis subsp. grosii (Pau) Devesa Casarabonela. 990 m. MGC 61974, 61909.
Lavandulo-Echinospartion boissieri.

Sideritis incana subsp. occidentalis (F. Quer) Cabezudo, J. M. Nieto \& T. Navarro Casarabonela. 990 m. MGC 61972, 61904.

Lavandulo-Echinospartion boissieri.

Silene andryalifolia Pomel

E1 Burgo. 1180 m. MGC 61950. Saxifragion camposii.

Sorbus aria (L.) Crantz subsp. aria

El Burgo. 1170 m. MGC 61923. AceriQuercion fagineae. NT

Sorghum halepense (L.) Pers.

Casarabonela. 1110 m. MGC 61966.

Stachys circinata L'Hér subsp. circinata

E1 Burgo. 1160 m. MGC 61929.

Stachydetum circinatae

Stipa offneri Breistr.

Alozaina. 1350 m. MGC 61929. Lavandulo-

Echinospartion boissieri.

Teucrium aureum subsp. angustifolium (Willk)

S. Puech

Casarabonela. 960 -1100 m. MGC 61913, 61906, 61915. Lavandulo-Echinospartion boissieri. NT

Teucrium bracteatum Desf.

Casarabonela. 1000 m. MGC 36262, 45247.

Rumicetalia indurati. EN

Teucrium chamaedrys Boiss.

Casarabonela. 1100 m. MGC 61912.

Lavandulo-Echinospartion boissieri.

Thymus $\mathrm{x}$ arundanus Willk.

E1 Burgo. 1100-1200 m. MGC 61947, 61930. Lavandulo-Echinospartion boissieri.

Thymus granatensis Boiss. subsp. granatensis

Casarabonela. 1070 m. MGC 61961, 61908.

E1 Burgo. 1250 m. MGC 61943. Andryalion agardhii. 


\section{BIBLIOGRAFÍA}

CABEZUDO, B., S. TALAVERA, G. BLANCA, C. SALAZAR, M. CUETO, B. VALDÉS, J. E. HERNÁNDEZ-BERMEJO, C. M. HERRERA, C. RODRÍGUEZ HIRALDO y D. NAVAS -2005- Lista roja de la flora vascular de Andalucía. Consejería de Medio Ambiente. Junta de Andalucía.

CEBALlOS, L. y C. VICIOSO -1933-Estudio sobre la vegetación y flora forestal de la provincia de Málaga. Inst. Forestal de Invest. y Exp. Madrid.

PÉREZ LATORRE, A. V., D. NAVAS, P. NAVAS, Y. GIL y B. CABEZUDO -1998.-Datos sobre la flora y la vegetación de la Serranía de Ronda (Málaga, España). Acta Bot. Malacitana 23: 149-191.
PÉREZ LATORRE, A. V., D. NAVAS FERNÁNDEZ, O. GAVIRA, G. CABALLERO y B. CABEZUDO -2004Vegetación del Parque Natural de las Sierras Tejeda, Almijara y Alhama (Málaga-Granada, España). Acta Bot. Malacitana 30: 117-190.

Aceptado para su publicación en septiembre de 2005

Dirección de los autores. Dpto. Biología Vegetal. Facultad de Ciencias. Universidad de Málaga. Campus de Teatinos, s/n. 29071, Málaga.

104. NUEVAS APORTACIONES AL CONOCIMIENTO DE LA FLORA DEL MACIZO DE LA SIERRA DE LÍBAR (MÁLAGA-CÁDIZ, PARQUES NATURALES SIERRA DE GRAZALEMA Y LOS ALCORNOCALES). III

\section{Manuel BECERRA PARRA}

New records for the flora of Sierra de Libar (Sierra de Grazalema and Los Alcornocales natural parks, Málaga and Cádiz provinces)

Palabras clave. Flora, Parque Natural, Sierra de Grazalema, Los Alcornocales, Málaga, Cádiz, Sierra de Líbar.

Key words. Flora, Natural Park, Sierra de Grazalema, Los Alcornocales, Málaga, Cádiz, Sierra de Líbar.

Durante los años 2004 y 2005 hemos continuado con nuestras herborizaciones en el Macizo de la Sierra de Líbar con el fin de completar el catálogo florístico de este conjunto montañoso situado entre las provincias de Málaga y Cádiz. Fruto de estas recolectas son las 24 citas nuevas para la Sierra de Líbar, de las cuales 6 lo son para el 